\title{
Kinetic Compensation Effect in the Thermal Decomposition of Biomass in Air Atmosphere
}

\author{
LIU Naian ${ }^{a}$ WANG Binghong ${ }^{b}$ FAN Weicheng ${ }^{a}$ \\ ${ }^{a}$ State Key Laboratory of Fire Science, University of Science and Technology of \\ China, Hefei, Anhui 230026, P. R. China \\ ${ }^{\mathrm{b}}$ Department of Modern Physics, University of Science and Technology of China, \\ Hefei, Anhui 230026, P. R. China
}

\begin{abstract}
The wood and leaf samples of eight species are examined by non-isothermal means to determine the mass loss kinetics of the thermal decomposition with linear temperature programming in air atmosphere. A simple kinetic description is developed based on the experimental results and integral analysis method. In the model, the mass loss process consists of three steps. The first step corresponds to the water evaporation, and the subsequent two mass loss steps are mainly due to two major pseudo components. The two pseudo components decompose respectively at two separate temperature regions. Under this kinetic scheme, the kinetic compensation effect is analyzed and it is found that the kinetic parameters $E$ and $A$ resulted from the variation of the species and the variation of model functions exhibit kinetic compensation effect. Quantitative and statistical criterion to distinguish between real and false compensation effect is discussed in detail.
\end{abstract}

KEY WORDS: cellulosic materials, thermal decomposition, kinetic modeling, kinetic compensation effect

\section{INTRODUCTION}

Biomass is frequently implicated in fire incidence. On the fire triangle of heat, fuel and oxygen, the rate of mass loss due to thermal decomposition determines the available fuel. To a lesser extent, the mass loss rate also determines the heat release rate, since the heat release rate in the fire can be modeled by the product of the heat of combustion and the mass of fuel burned. Understanding the thermal decomposition of biomass and at the same time obtaining the kinetic parameters are of great importance for the modeling of biomass combustion and fire propagation and thermochemical conversion processes.

During the past several decades, it has been found that for the kinetic parameters, i.e. the pre-exponential factor $A$ and the activation energy $E$, kinetic investigations of heterogeneous solid-gas decompositions often reveal the validity of the following relationship between $E$ and $A$ :

$$
\ln A=a E+b
$$

where $a$ and $b$ are constant coefficients for a series of related rate process. This relationship is referred to as the "kinetic compensation effect (KCE)", and here the so-called "compensation" means that the reduction in rate which is expected to result from an increase in activation energy does not occur for the set of reactions obeying Eq. 
(1), due to a compensatory increase of $A$. In other words, it reflects a compensation effect between the exponential and pre-exponential factors in the Arrhenius equation.

In traditional kinetic studies involving homogeneous reactions, the evaluation of the kinetic parameters assumed importance because they were considered to be indicative of the reaction mechanism. However, in the case of heterogeneous reactions taking place in the solid state, both these parameters lose their relevance because the concepts of "order of reaction" and "concentration" are not applicable. Hence, $E$ and $A$ values have little physical significance. It is also evident from the literature that the value of $E$ depends heavily on various experimental factors such as sample size, particle size and its distribution, heating rate, presence of impurities in the sample, gaseous atmosphere in and around the sample etc. Thus we may ask whether the experimentally determined Arrihenius parameters have any relevance to their practical application. The KCE in fact provides a possible means to predict the effects of experimental factors on kinetic parameters. According to this relationship, for any change in the experimental activation energy arising from the variation of experimental conditions, a corresponding change in $A$ also occurs, thus we could correlate the different parameters under different experimental conditions [1]. True KCE can prove to be useful in chemical research for identifying the governing reaction mechanism; predicting effects of various parameters on reactions; predicting Arrhenius parameters when limited data is available; separating the effects of surface and bulk properties; and optimizing process design [2].

The purpose of this paper is to examine the $\mathrm{KCE}$ relationship existing in the thermal decomposition of biomass materials. For biomass decomposition, the KCE relationship, if available, would help correlate the kinetic parameters under different experimental conditions, and thus be useful for the modeling of biomass combustion. We emphasize on the $\mathrm{KCE}$ resulted from the species variation and model variation. The criterion to recognize a real KCE relationship is also investigated.

\section{EXPERIMENTAL}

The raw materials used in the investigation were respectively the wood and leaf of fir, banana shrub, tea tree, waxberry, holm oak, heath, masson pine and nanmao collected from Qimen forest zone of China (Table 1). These materials were first cut and then ground, thereby the average particle size was specified to be approximately $40 \mu \mathrm{m}$. The grains of the sample were evenly distributed over the open sample pan of $5 \mathrm{~mm}$ diameter, loosely, with the initial amounts of the samples all kept to be $10 \mathrm{mg}$ or so. The depth of the sample layer filled in the pan was about $0.5 \mathrm{~mm}$. Thermal decomposition was observed in terms of the overall mass loss by using a STA 409C Thermobalance. Temperature calibration of TGA was carried out with special concern, since in this device the thermocouple was not in direct contact with the sample. An air stream was continuously passed into the furnace at a flow rate of $60 \mathrm{ml} / \mathrm{min}$ (at normal temperature and atmospheric pressure). The temperature was increased to $750^{\circ} \mathrm{C}$ at a rate of $10^{\circ} \mathrm{C} / \mathrm{min}$. The heating rate of this order is generally considered able to ensure that no temperature gap exists between the sample and its surroundings [3]. In order to test the transport effect under this heating rate and sample mass condition, we especially carried out the experiments using the masson pine as an example with different sample masses for which the least being $4 \mathrm{mg}$ and the most being $10.6 \mathrm{mg}$. The result indicated that the 


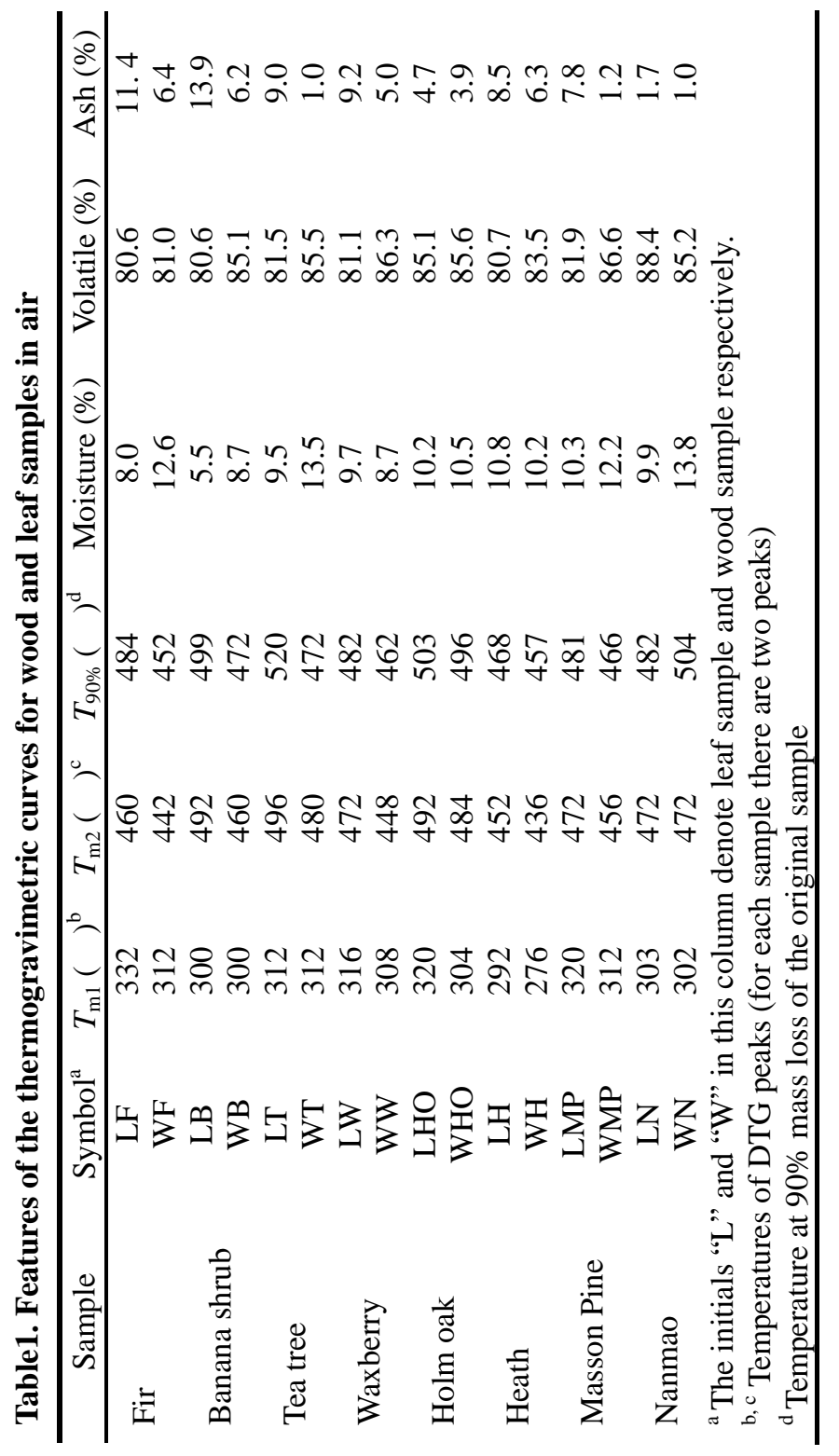


TG curves for different masses differed from each other only around the upper edge of the domain of evaluation, at temperatures starting around $500^{\circ} \mathrm{C}$. We regarded one of the curves as a benchmark curve and evaluated the fit of any other curve (e.g., the $j$ th curve) relative to this benchmark curve according to the following expression:

$$
\operatorname{fit}(\%)=\left(\sum_{i=1}^{N_{j}}\left[w_{j i}-w_{b i}\right]^{2} / N_{j}\right)^{0.5}
$$

where $w$ is the mass percentage, the subscript $b$ refers to the benchmark curve, and $N_{j}$ is the number of points on the $j$ th curve. Acceptable agreements were achieved with the worst fit being only 2.6, indicating that the effect of the transport processes is low. This may be partly due to the low heating rate employed in this study. The sample mass loss percentage and its temperature were recorded continuously as a function of heating time. From the sample mass-loss percentage, the normalized mass-loss ratio of a sample can be determined and plotted versus the sample temperature as the TG curve. The DTG curve can then be calculated by differentiating the mass-loss ratio with respect to time or temperature (Fig. 1).

\section{RESULTS AND DISCUSSION}

\section{Kinetic Model of the Mass loss Process}

Following the water evaporation, two distinct DTG peaks are observed for all the samples subjected to experiments in air. When the temperature reaches relatively high, oxidation of the char residue generated in the early stage is likely to occur. The first DTG peak occurs at about $300^{\circ} \mathrm{C}$. Comparing the present results with those in literature [4-6] (at the same or almost the same heating rates but in nitrogen atmosphere) shows that the present first DTG peak temperature is close to that in literature, indicating that the first major mass loss is little affected by oxidation of char. From this evidence it can be concluded that the mechanism controlling this step is mainly due to the pyrolysis of hemicellulose and cellulose and partly due to lignin pyrolysis which, however, occurs in a broad temperature range generally. For different sample species, the temperature of the second DTG peak varies between $440-500^{\circ} \mathrm{C}$, which is much higher than that in nitrogen atmosphere [6], implying that the second major mass loss is ascribed to the combined effect of lignin pyrolysis and char oxidation.

The experimental results show that the two DTG peaks appear nearly separate in almost all the cases, indicating that the two controlling mechanisms illustrated above interact little with each other, and they take effect respectively in the lower and higher temperature ranges. This evidence leads to the basic assumption of the kinetic model, i.e. the two major mass losses can be regarded as due to two independent reactions of two pseudo components occurring respectively in the lower and higher temperature ranges. Correspondingly, the initial and residue solid mass fractions for the two separate reactions are defined respectively in the definite lower and higher temperature ranges, and the temperature corresponding to the minimum in the DTG curve is regarded as the point of separation between the two temperature ranges. Hereby the basic equations of the kinetic model can be expressed as follows 

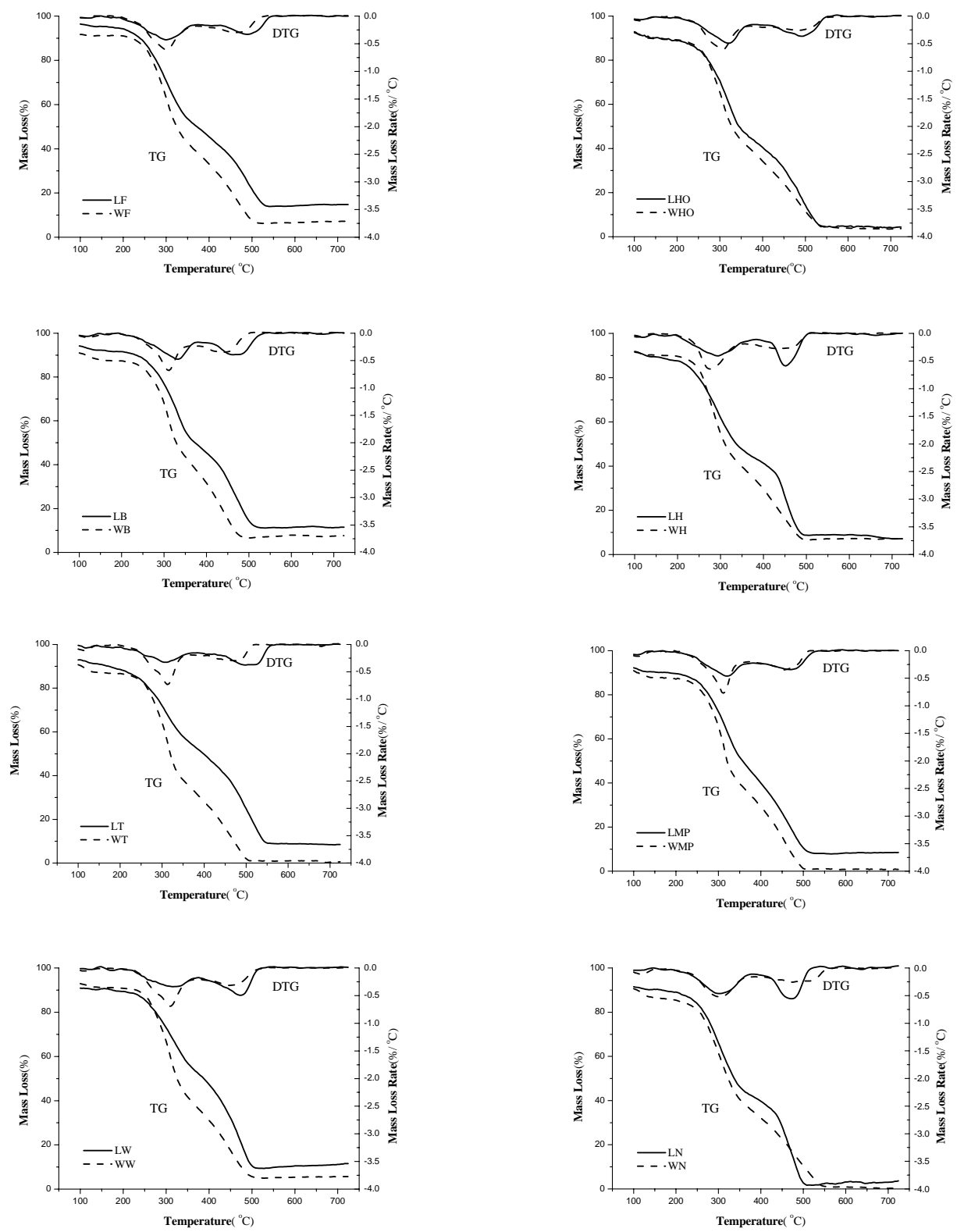

Fig. 1 - Experimental TG and DTG curves for wood ( - - - ) and leaf $(-)$ samples. 


$$
\begin{gathered}
\frac{\mathrm{d} \alpha}{\mathrm{d} T}=\left\{\begin{array}{lc}
\frac{w_{10}-w_{1 \infty}}{w_{10}-w_{2 \infty}} \frac{\mathrm{d} \alpha_{1}}{\mathrm{~d} T} & T_{10}<T<T_{1 \infty} \\
\frac{w_{20}-w_{2 \infty}}{w_{10}-w_{2 \infty}} \frac{\mathrm{d} \alpha_{2}}{\mathrm{~d} T} & T_{1 \infty}=T_{20}<T<T_{2 \infty}
\end{array}\right. \\
\text { in which }\left\{\begin{array}{lr}
\frac{\mathrm{d} \alpha_{1}}{\mathrm{~d} T}=\frac{A_{1}}{\beta} \exp \left(-E_{1} / R T\right) f_{1}\left(\alpha_{1}\right) & T_{10}<T<T_{1 \infty} \\
\frac{\mathrm{d} \alpha_{2}}{\mathrm{~d} T}=\frac{A_{2}}{\beta} \exp \left(-E_{2} / R T\right) f_{2}\left(\alpha_{2}\right) & T_{1 \infty}=T_{20}<T<T_{2 \infty}
\end{array}\right.
\end{gathered}
$$
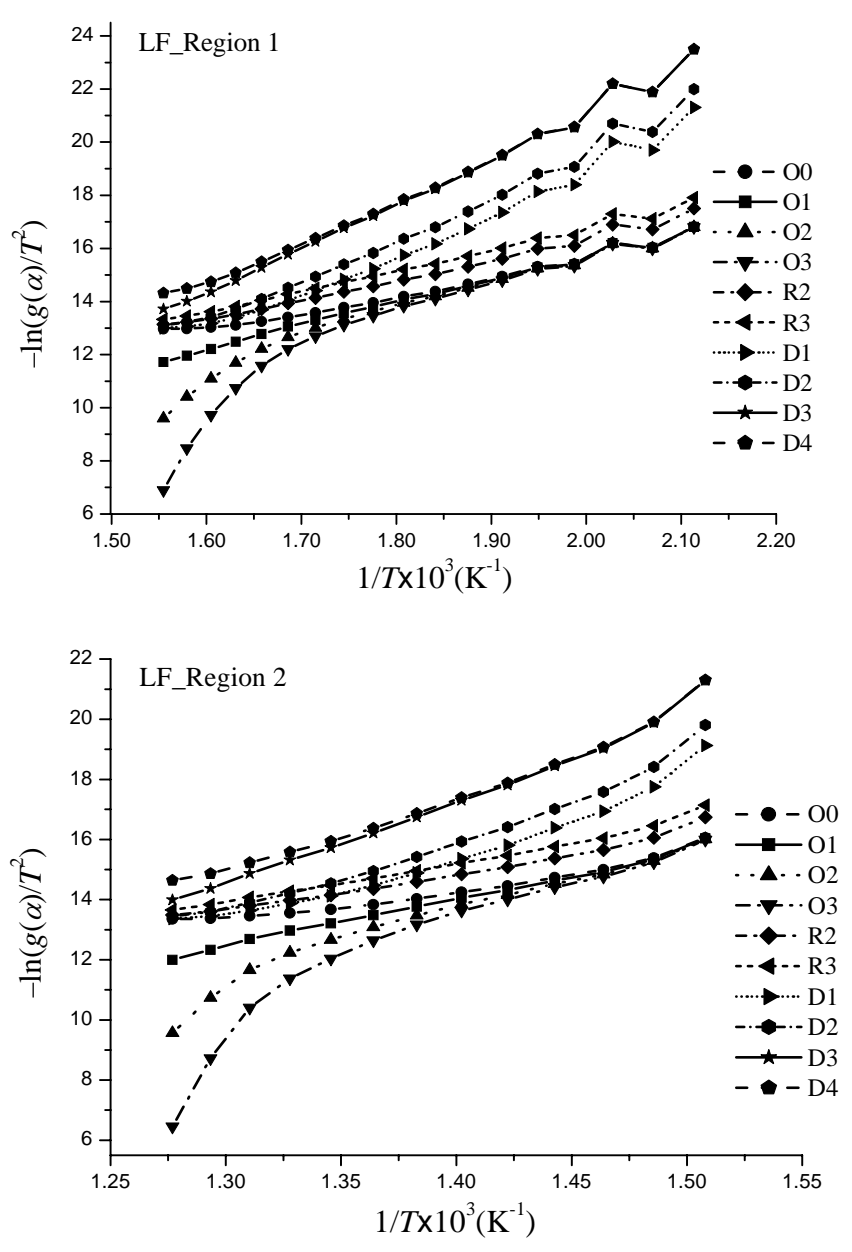

Fig. 2 - Plot of $\ln \left[g(\alpha) / T^{2}\right] \sim 1 / T$ for the sample of LF. Here O0, O1, O2, O3, R2, R3, D1, D2, D3, D4 represent different model functions as indicated in Table 2. where the subscripts 1 and 2 respectively refer to the two pseudo components 1 and 2, which react respectively in the lower and higher temperature ranges. The variable $\alpha$ is the degree of transformation of the sample itself defined globally, while $\alpha_{1}$ and $\alpha_{2}$ refer to the transformation of the components 1 and 2 respectively in the two temperature ranges. $R$ is the ideal gas constant $\left(8.314 \times 10^{-3} \mathrm{~kJ} \quad \mathrm{~mol}^{-1} \mathrm{~K}^{-1}\right)$. The parameter $\beta$ is heating rate $\left({ }^{\circ} \mathrm{C} / \mathrm{min}\right), T$ is the absolute temperature of the sample, and $w$ is the mass fraction of the sample itself. The subscripts 0 and $\infty$ refer to the initial and residual amounts of the raw component, respectively. $T_{1 \infty}$ (also namely $T_{20}$ ) is the point of separation between the two temperature ranges.

Here we use the integral Coats-Redfern method [7] to perform the kinetic analysis for the TG curves, and the basic equation of this method for component $\mathrm{i}$ 
$(\mathrm{i}=1,2)$ is

$$
\ln \left[\frac{g_{\mathrm{i}}\left(\alpha_{\mathrm{i}}\right)}{T^{2}}\right]=\ln \left\{\frac{A_{\mathrm{i}} R}{\beta E_{\mathrm{i}}}\left[1-\frac{2 R T}{E_{\mathrm{i}}}\right]\right\}-\frac{E_{\mathrm{i}}}{R T}
$$

where $g_{\mathrm{i}}\left(\alpha_{\mathrm{i}}\right)$ is the integral of the reciprocal of $f_{\mathrm{i}}\left(\alpha_{\mathrm{i}}\right)$ between $0 \sim \alpha_{\mathrm{i}}$. Obviously, the key of this method is the determination of the correct form of $f_{\mathrm{i}}\left(\alpha_{\mathrm{i}}\right)(\mathrm{i}=1,2)$ by trial. Based on the correct form of $f_{\mathrm{i}}\left(\alpha_{\mathrm{i}}\right)(\mathrm{i}=1,2)$, the activation energy and pre-exponential factor can be determined respectively from the slope and intercept terms of the regression line.

The calculation results show that the first order function (O1) leads to good linearity with regard to Eq. (5) for all the wood and leaf samples without exception. Taking the leaf sample of fir (LF) as an example, with 10 different functions in Table 2 used, Fig. 2 shows the plots of $\ln \left[g_{\mathrm{i}}\left(\alpha_{\mathrm{i}}\right) / T^{2}\right] \sim 1 / T$ with the kinetic scheme. By observation and the comparison of the correlation coefficients, it can be verified that the $\mathrm{O} 1$ function leads to the best linearity for both components 1 and 2. Similar plots are obtained for other samples, and the presentation of these plots is omitted here because of space constraint. It can be seen from Fig. 2 that for the model functions based on "order" of reaction, the plots for the functions of $\mathrm{O} 2$ and $\mathrm{O} 3$ bend down greatly, while the plot for $\mathrm{O} 0$ bends up slightly. It is the function of $\mathrm{O} 1$ that shows the best linearity. Therefore the kinetic parameters obtained by $\mathrm{O} 0, \mathrm{O} 2$ and $\mathrm{O} 3$ have no kinetic significance. However, as to be clarified in the later section, $\mathrm{KCE}$ can be revealed from these "pseudo" kinetic parameters.

\section{Kinetic compensation effect (KCE) of the kinetic parameter}

$\mathrm{KCE}$ is often correlated with the concept of Isokinetic Point (IKP). IKP refers to a common point of intersection of Arrhenius lines (i.e. $\ln k(T)$ versus $T^{-1}$ ). From the Arrhenius equation we obtain

$$
\ln A=\frac{1}{R T} E+\ln k
$$

Comparing this expression with (1), we can see that for all the rate processes whose kinetic parameters are in the parameter set which satisfies (1), the corresponding Arrhenius lines have a common point of intersection $\left(T_{\text {iso }}{ }^{-1}, \ln k_{\text {iso }}\right)$ :

$$
\begin{aligned}
& T_{\text {iso }}=\frac{1}{R a} \\
& \ln k_{\text {iso }}=b
\end{aligned}
$$

Eq. (1) can thus been rewritten as

$$
\ln A=\frac{1}{R T_{\text {iso }}} E+\ln k_{\text {iso }}
$$

Using the kinetic parameters calculated for the model function O1, we obtain the plot 
$\ln A \sim E$ respectively corresponding to the two pseudo components, as shown in Fig. 3. The kinetic parameters $E$ and $A$ are indicated to satisfy the KCE relationship, and the two linear relations are respectively:

$$
\text { Component } 1 \quad \ln A=-3.0334( \pm 0.3724)+0.2262( \pm 0.0048) E \quad r=0.9968
$$

Component $2 \quad \ln A=-2.5444( \pm 0.5375)+0.1727( \pm 0.0043) E \quad r=0.9957$

where $r$ is the correlation coefficient. $E$ is expressed in $\mathrm{kJ} / \mathrm{mol}$ and $A$ in $\mathrm{min}^{-1}$. The figures

Table 2. Kinetic model functions $f(\alpha)$ and corresponding $g(\alpha)$ usually employed for the solid state reactions

\begin{tabular}{|c|c|c|}
\hline Model & $g(\alpha)=k t$ & $f(\alpha)=(1 / k)(d \alpha / d t)$ \\
\hline \multicolumn{3}{|c|}{ Reaction order } \\
\hline $\mathrm{O} 0$ & $\alpha$ & 1 \\
\hline $\mathrm{O} 1$ & $-\ln (1-\alpha)$ & $1-\alpha$ \\
\hline $\mathrm{O} 2$ & $(1-\alpha)^{-1}$ & $(1-\alpha)^{2}$ \\
\hline $\mathrm{O} 3$ & $(1-\alpha)^{-2}$ & $(1-\alpha)^{3}$ \\
\hline \multicolumn{3}{|c|}{ Phase boundary controlled reaction } \\
\hline $\mathrm{R} 2$ & $1-(1-\alpha)^{1 / 2}$ & $2(1-\alpha)^{1 / 2}$ \\
\hline R3 & $1-(1-\alpha)^{1 / 3}$ & $3(1-\alpha)^{2 / 3}$ \\
\hline \multicolumn{3}{|c|}{ Diffusion } \\
\hline D1 & $\alpha^{2}$ & $1 / 2 \alpha$ \\
\hline D2 & $(1-\alpha) \ln (1-\alpha)+\alpha$ & {$[-\ln (1-\alpha)]^{-1}$} \\
\hline D3 & {$\left[1-(1-\alpha)^{1 / 3}\right]^{2}$} & $3 / 2(1-\alpha)^{2 / 3}\left[1-(1-\alpha)^{1 / 3}\right]^{-1}$ \\
\hline D4 & $(1-2 \alpha / 3)-(1-\alpha)^{2 / 3}$ & $3 / 2\left[(1-\alpha)^{-1 / 3}-1\right]^{-1}$ \\
\hline
\end{tabular}

in brackets represent errors limits at $95 \%$ confidence for the reported parameters. As indicated above, many factors variation can lead to the KCE relationship. However, the KCE relationship due to species variation has rarely been reported so far. It should be noted that in the study of the pyrolysis of pitch, a similar KCE relationship was reported [8]. The author used four kinds of pitch materials, and at the same time adopted the Ozawa method, Friedman method, and Doyle method to calculate the kinetic parameters. The kinetic parameters due to the species and method variations were found to satisfy a good KCE relationship.

As we know, although the true energy barrier may be related to the calculated value of $E$ in the solid state reaction kinetics, its true relationship will not be clear until a definition or the significance for a "mole of solid" is established. Hence, in order to avoid confusion and to distinguish the difference between gas phase and solid state reactions, $A$ should be called the pre-exponential factor rather than the frequency factor, and $E$ should be called the apparent activation energy. However, from the present analysis we can see that the two pseudo components respectively satisfy two distinct $\mathrm{KCE}$ relations, which is consistent with the fact that the mass losses in these two temperature regions are respectively controlled by two different kinetic processes. This implies that KCE relations, although based on apparent kinetic parameters, to some degree reveal the reaction mechanism. The $\mathrm{KCE}$ relation to some degree provides a clue to recognize whether or not the obtained parameter set $(E, A)$ fit the same mass loss kinetics: If two 
parameter sets $(E, A)$ satisfy two different $\mathrm{KCE}$ relations, then different kinetics may be correlated with them.

Then, if two sets $(E, A)$ satisfy the same KCE relation, can it be concluded that they satisfy the same kinetic expressions? The answer seems to be No. We shall clarify this point in the following paragraphs.

Although the model function $\mathrm{O} 1$ has been verified to be the most reasonable kinetic description of the biomass decomposition in this study, "Pseudo" kinetic parameters can be calculated by different reaction orders. The plots of $\ln A \sim E$ for all the samples are indicated in Fig. 4. It's obvious that for the two pseudo components, very high correlation coefficients of the plots are achieved. The regression straight lines are respectively

Region 1: $\ln A=-3.4943( \pm 0.1364)+0.2327( \pm 0.0014) E \quad r=0.9978$

Region 2: $\ln A=-2.8534( \pm 0.2116)+0.1727( \pm 0.0011) E \quad r=0.9976$

This KCE is derived from the model variation, and so it is believed to be lack of chemical significance. In this sense it can be called "pseudo KCE". The implication of this "pseudo KCE" has not yet been realized. However, It can be concluded that, when different parameter sets $(E, A)$ are found to satisfy a specific KCE relation, it's still possible that they support different kinetic descriptions.

\subsection{On the criterion to distinguish between true and false kinetic compensation effect}

As clarified earlier, the $\mathrm{KCE}$ relation is in fact equivalent to the so called isokinetic effect. In the coordinate of $\ln k-1 / T$, the straight lines corresponding to different kinetic

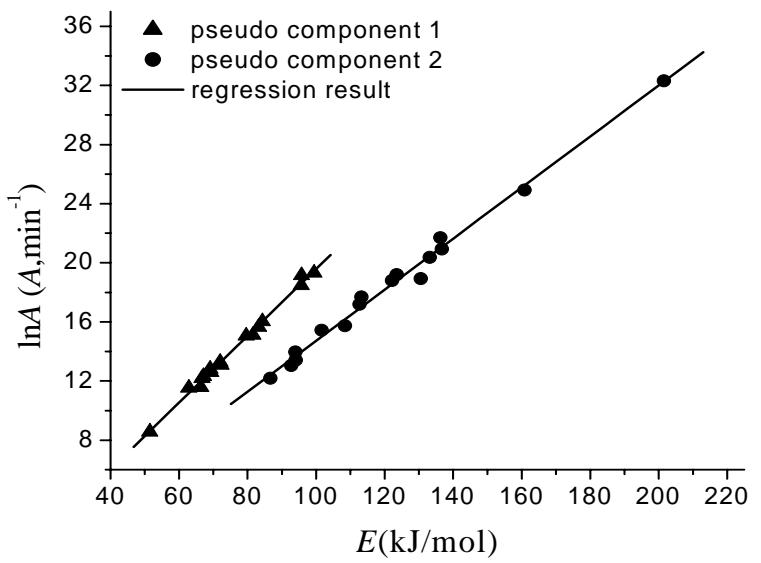

Fig. 3 - Compensation plot of kinetic parameters for all samples (due to species variation). parameters $(A, E)$ which satisfying $\mathrm{KCE}$ relation have a common interaction point $\left(1 / T_{\text {iso }}, \ln k_{\text {iso }}\right)$. Agrawal [9] claimed a criterion to recognize the $\mathrm{KCE}$ relation, i.e. $\mathrm{KCE}$ relation exists only if the plots $\ln k \sim 1 / T$ display concurrence at a single point. If a series of reaction displays the linear plot of $\ln A \sim E$ with relatively high correlation coefficient, but fails to display a single point of concurrence, then the system exhibits a false compensation effect. Agrawal used a few examples in literature to justify his viewpoint. This

criterion is correct in theory, but is obviously too restrictive to meet. Later, Agrawal did not persist in his original rigorous procedures. In one of his papers [2] he clarified that in 
actual experimental data, there will be experimental errors in measuring temperature $T$ and computational errors in determining the reaction rate constant $k$. Due to these errors, even if the system exhibits a true $\mathrm{KCE}$, a single point of concurrence may not be observed. Instead the rates should at least appear to converge at $T_{\text {iso. }}$. But in the face of so

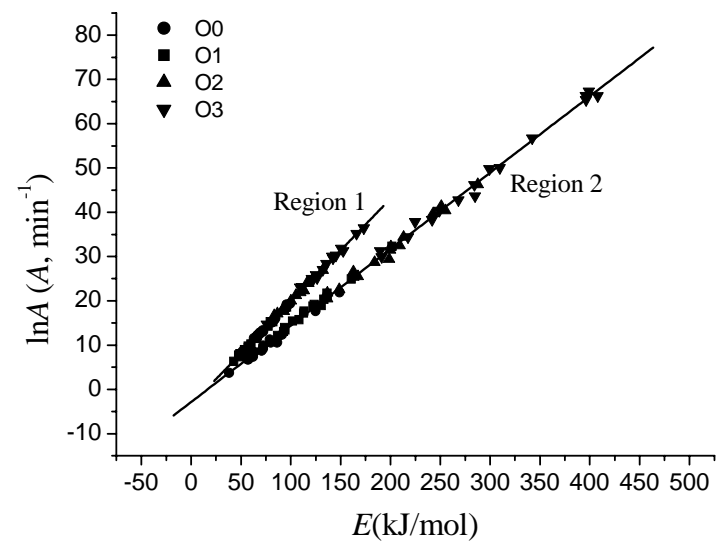

Fig. 4 - Compensation plot of kinetic parameters for all samples and reaction order models $(n=0,1,2,3)$ (due to species variation and model simultaneously). many straight lines, how can we judge whether or not they have a trend to converge at a single point quantitatively? This is to be clarified as follows.

As indicated earlier, for the lower or high temperature range, the kinetic parameters for different order functions indicate linear relations between $\ln A$ and $E$. Here and in the following discussion we use the mass loss process of the pseudo component 1 which occur in the lower temperature range as the example to illustrate our point. Fig. 5 indicates the plot of $\ln k$ versus $T^{1}$ for all the kinetic parameters for different order functions. Obviously these straight lines don't have a common point of intersection, however they tend to converge at a single point. We now define a measure to characterize this tendency quantitatively.

As we know, whether or not the effect is a real or false compensation effect, the $\ln k_{\text {iso }}$ and $1 / T_{\text {iso }}$ can be proximately evaluated from Expression (11). For each straight line in

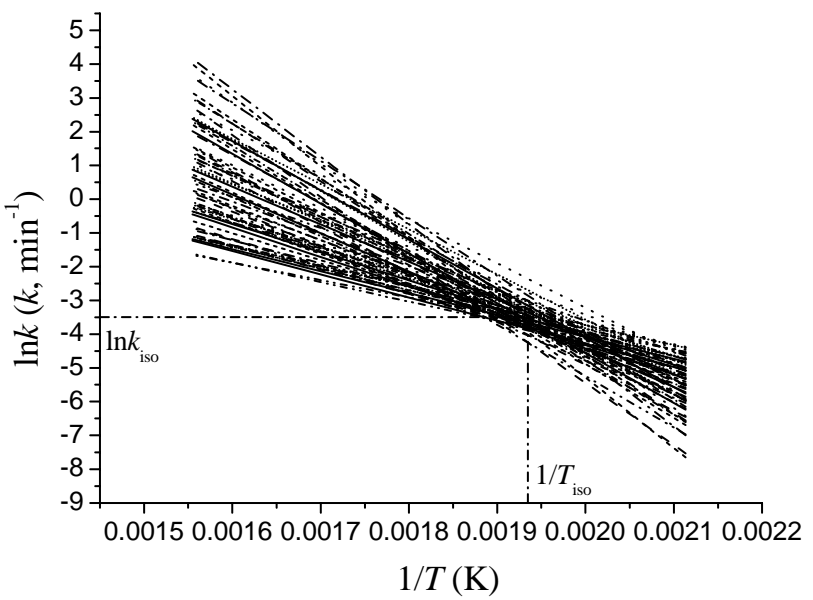

Fig. 5 - Plot of $\ln k$ versus $T^{1}$ for all the samples and reaction order models $(n=0,1,2,3)$ (due to species variation and model variation simultaneously).
Fig. 5, $\left.\quad(\ln k)\right|_{T=T_{\text {iso }}}$ can be calculated. In fact, $\left.(\ln k)\right|_{T=T_{\text {iso }}}$ can be looked at on as a random variable since the straight lines in Fig. 5 are independent of each other. The value of $\ln k_{\text {iso }}$ can be looked on as the predicted value of $\left.(\ln k)\right|_{T=T_{\text {iso }}}$. Thus all the $\left.(\ln k)\right|_{T=T_{\text {iso }}}$ values obtained can be looked as the random samples. When the number of samples is relatively high (generally higher than 50), 
the mean value of the samples can be used as the estimation. If the estimation of $\left.(\ln k)\right|_{T=T_{\text {iso }}}$ prove to be close to $\ln k_{\text {iso }}$, then the compensation effect obtained are recognized to be a true KCE.

By the above procedure we obtain that the mean value of $\left.(\ln k)\right|_{T=T_{i s o}}$ is -3.4929 , which is very close to -3.4943 as indicated in Expression (11), and the standard deviation of the mean value is 0.04 . This result indicates that the KCE obtained in this paper is a real compensation effect, although the implication of the so-called "pseudo compensation effect" due to model variation is not yet clear.

\section{CONCLUSIONS}

- In this paper, the model function of first order gives the best fits to the experimental data of the thermal decomposition of biomass compared with other model functions. This result suggests the possibility that "first order" reflects the real mechanism. However at present there is no direct evidence that this is so, and we merely regard this rate law as providing the best engineering fit to the data.

- The kinetic parameters $E$ and $A$ of biomass decomposition derived from the species variation the variation of model functions exhibit the kinetic compensation effect. When different parameter sets $(E, A)$ are found to satisfy a specific KCE relation, it's still possible that they support different kinetic descriptions.

- The $\left.(\ln k)\right|_{T=T_{i s o}}$ can be used a random variable to help distinguish between real and false compensation effect Quantitatively by statistical means.

\section{NOMENCLATURE}

$A=$ apparent pre-exponential factor $\left(\mathrm{min}^{-1}\right)$

$E=$ apparent activation energy $(\mathrm{kJ} / \mathrm{mol})$

$n=$ apparent reaction order

$r=$ correlation coefficient

$R=$ gas constant $\left(\mathrm{kJ} \cdot \mathrm{K}^{-1} \cdot \mathrm{mol}^{-1}\right)$

$T=$ absolute temperature $(\mathrm{K})$

$T_{0}=$ initial decomposition temperature $(\mathrm{K})$

$T_{\mathrm{m} 1}, T_{\mathrm{m} 2}=$ the temperatures of the DTG peaks $(\mathrm{K})$

$T_{\mathrm{f}}=$ final decomposition temperature $(\mathrm{K})$

$w=$ sample mass percentage

$w_{0}=$ initial sample mass percentage

$w_{\infty}=$ residual sample mass percentage

$\alpha=$ mass loss fraction

$\beta=$ heating rate $(\mathrm{K} / \mathrm{min})$

\section{ACKNOWLEDGEMENTS}


This work was sponsored by National Natural Science Foundation of China under Grants 59936140, the China NKBRSF project (No. 2001CB409600), and the Anhui Excellent Youth Scientist Fundation (2001-2002). This study was also supported by USTC Youth Fundation and National Key Technologies R\&D Programme (2001BA510B09-03). N. A. Liu was supported by the China-Greece Joint Project "Investigation on the Characteristics of Forest Fire in the Early Stage and Its Control Technique" and Natural Science Foundation of Anhui Province (00047416) .

\section{LITERATURE}

[1] Prasad, T. P., Kanungo, S. B., Ray, H. S., "Non-isothermal kinetics: some merits and limitations," Thermochimica Acta 203: 503-514 (1992).

[2] Agrawal, R. K., "The compensation effect. A reply to Zsako and Somasekharan's remarks," Journal of Thermal Analysis 34: 1141-1149 (1988).

[3] Bilbao, R., Mastral, J. F., Aldea, M. E., Ceamanos, J., "Kinetic study for the thermal decomposition of cellulose and pine sawdust in an air atmosphere," Journal of Analytical and Applied Pyrolysis 39: 53-64 (1997).

[4] Orfao, J. J. M., Antunes, F. J. A., Figueiredo, J. L., "Pyrolysis kinetics of lignocellulose materials-three independent reactions model," Fuel 78: 349-358 (1999).

[5] Caballero, J. A., Font, R., Marcilla, A., "Comparative study of the pyrolysis of almond shells and their fractions, holocellulose and lignin. Product yields and kinetics," Thermochimica Acta 276: 57-77 (1996).

[6] Font, R., Marcilla, A., Verdu, E., Devesa, J., "Thermogravimetric kinetic study of the pyrolysis of almond shells and almond shells impregnated with $\mathrm{CoCl} 2$," Journal of Analytical and Applied Pyrolysis 21: 249-264 (1991).

[7] Coats, A. W., Redfern, J. P., "Kinetic parameters from thermogravimetric data," Nature 201: 68-69 (1964).

[8] Collett, G. W., Rand, B., "Thermogravimetric investigation of the pyrolysis of pitch materials. A compensation effect and variation in kinetic parameters with heating rate," Thermochimica Acta 41:153-165 (1980).

[9] Agrawal, R. K., "On the compensation effect," Journal of Thermal Analysis 31:73-86 (1986). 